\title{
LATEX GLOVES - IS ALLERGY A PROBLEM?
}

\section{A Sinha, Associate Specialist, Orthopaedics Department; Ros Schofield, Clinical Audit Officer; S Westbrook, Senior Orthopaedic Nurse; PV Harrison, Consultant Dermatologist, Lancaster Acute NHS Trust}

\section{INTRODUCTION}

The latex glove is a primary barrier for protection against infection for healthcare personnel and its use has increased, particularly due to concerns about AIDS and hepatitis. Indeed, the Project 2000 nurse during training comes prepared onto the ward with gloves at the ready. The Occupational Safety and Health Administration in the USA estimated that more than 5.6 million American healthcare and other workers use latex gloves regularly, amounting to over seven billion pairs of gloves a year ${ }^{(1)}$.

Natural latex is produced by the Havea brasiliensis tree. The cloudy white liquid is collected by tapping the tree. The liquid then undergoes a complex coagulation process, which involves the addition of sulphur and organic chemicals. Natural rubber latex is composed, therefore, of natural proteins and added chemicals. These proteins cannot be completely removed during the manufacturing process. The recent increasing demand for latex gloves and other rubber products such as condoms has resulted in less refined products containing high concentrations of latex allergens ${ }^{(2)}$, possibly because of accelerated manufacturing processes. There is also considerable variation in the total protein and therefore allergen content of different brands of latex gloves $^{(3)}$. Apart from the gloves, rubber latex is also used to produce a range of other medical devices including catheters, condoms, elasticated bandages and wound drains.

As the frequency of use and duration of wearing latex gloves has increased, latex sensitisation has emerged as an increasing problem. We have investigated the extent of the problem amongst local health workers and a detailed questionnaire was distributed amongst all doctors, nurses, radiographers and pathology staff of the Royal Lancaster Infirmary. Questions were directed specifically towards the possibility of allergy from the use of gloves.

\section{MATERIALS AND METHODS}

Out of the total number of staff in Lancaster Acute NHS Trust ( 1899), 1313 staff were targeted for the study. Out of these, 301 returned the questionnaires (Table 1). The age range was $20-50$, mean 30 . There were 14 doctors, 66 nurses, 8 pathology staff, four radiographers and four clerical staff/ward clerks (Figure 1). The doctors included three surgeons, 5 physicians or paediatricians, two anaesthetists, two casualty medical officers, one haematologist and one radiologist. The nursing staff included 41 ward nurses 10 from intensive care units, eight from accident and emergency, four working in operating theatres, two from outpatient departments and one from the community (Figure 2).

\begin{tabular}{|lc|}
\hline Total number of staff in Acute Trust & 1899 \\
\hline $\begin{array}{l}\text { Total number of staff targeted for study } \\
\text { (doctors, nurses, pathology and radiology } \\
\text { staff and clerical staff) }\end{array}$ & 1313 \\
\hline Number of questionnaires returned & 301 \\
\hline Number of staff with possible latex allergy & $96(7.3 \%)$ \\
\hline
\end{tabular}

Table 1 - Incidence of latex glove allergy

\section{RESULTS}

Three hundred and one members of staff returned completed questionnaires. Of this group 96 (7.3\% of all staff) had symptoms highly suggestive of the possibility of latex glove allergy. Eighteen members of staff had had problems in the past which had settled, but the rest (78 staff members) continued to suffer from the problem. The doctors had

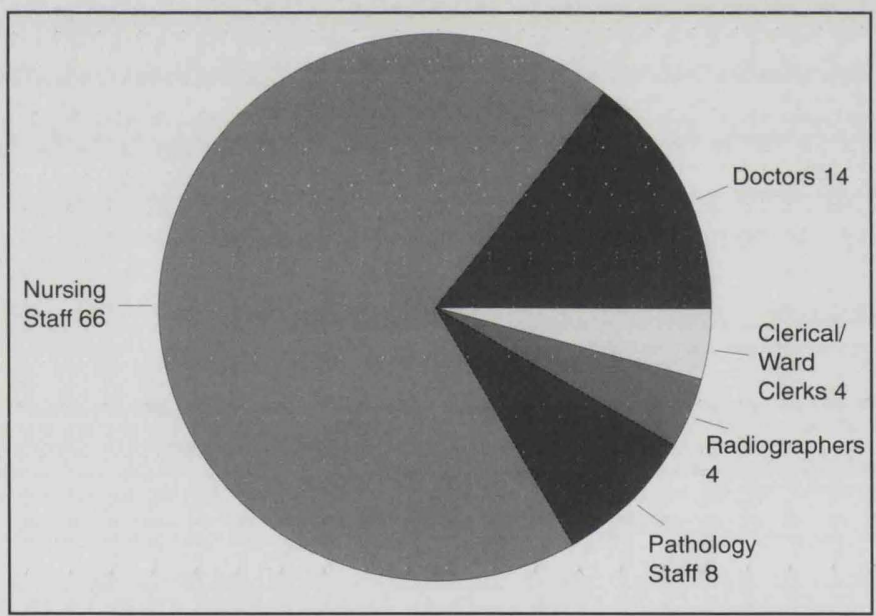

Fig. 1 -Distribution of suspected latex glove allergy amongst hospital staff

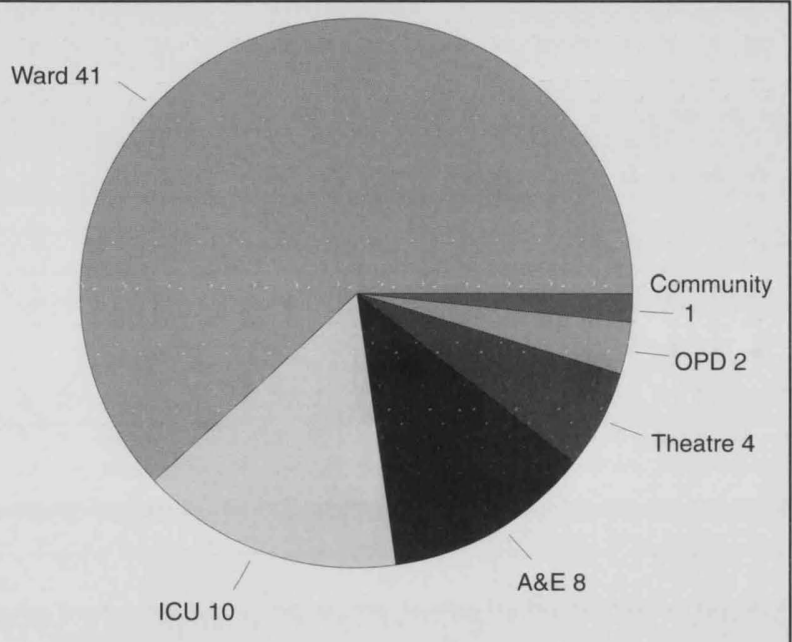

Fig. 2 -Distribution of suspected latex glove allergy among nurses 
experienced symptoms from between one and six years (median 3.5 years). Some nursing staff had noticed symptoms within six months of starting training whereas others had had problems for almost 20 years (median 3.5 years). The commonest symptom affecting the hands (itching) was noticed in 82 out of 96 individuals $(85 \%)$. Redness of the skin was noticed in 77 out of 96 individuals (80\%), and skin eruptions in 32 out of $96(33 \%)$. Skin scaling was noticed in only eight out of $96(8 \%)$ and one person complained of dry or cracking skin. One individual described an occasional burning sensation. Eleven out of 96 ( $12 \%$ ) experienced eye irritation and other symptoms including sneezing, nasal congestion and sometimes coughing. Eighty-five individuals $(89 \%)$ noticed that the symptoms were transient in nature but 11 had almost permanent problems. Ten individuals $(9.6 \%)$ indicated the problem affected their lifestyle but only one person needed time off work because of the problem.

Fifteen individuals commented that there appeared to be more of a problem with powdered gloves than unpowdered ones and ten indicated that there was not enough choice of surgical gloves available in the hospitals. Some people noticed that cheaper gloves were a greater problem than more expensive ones. Thirty-seven individuals (39\%) indicated that they had not realised that glove allergy could be a problem and 67 people $(70 \%)$ did not know what would be the most appropriate procedure to follow if they had suspicions of latex glove allergy. Eighty-four people $(88 \%)$ agreed that there should be a hospital policy regarding the use of gloves.

Fifty-seven $(60 \%)$ used some form of moisturising cream on their hands, and $42(44 \%)$ had endeavoured to identify hypoallergenic gloves. Twenty-two people (23\%) had consulted their general practitioners concerning the problem but only three had been to the occupational health department. Nine people had previously had patch testing to ascertain allergy and in each of them latex allergy was confirmed. Twenty individuals $(21 \%)$ indicated that using hypoallergenic gloves had solved the problem completely, but $53(55 \%)$ had only partial diminution of symptoms and seven had no change. Another 16 did not indicate whether using alternative gloves had helped their skin problem.

\section{DISCUSSION}

Increased use of gloves in the healthcare environment over the last few years has led to the growing incidence of latex allergy, now recognised in some countries. A study in the USA found that $17 \%$ of 224 self-selected hospital employees had positive evidence of latex sensitivity on allergy testing ${ }^{(4)}$. The highest incidence in Europe has been reported from Marseilles, where skin tests for latex sensitivity were positive in $10.7 \%$ of a group of 197 operating theatre nurses ${ }^{(5)}$. In another study from France $2.65 \%$ out of 907 hospital employees were found to be allergic to latex ${ }^{(6)}$. In Finland, an incidence of $3 \%$ latex allergy was reported amongst 512 hospital staff $^{(7)}$ and in a Swedish study examining 202 hospital and dental workers, $3.5 \%$ of the staff had latex sensitivity confirmed by testing ${ }^{(8)}$.

The medical device agency (MDA) has been monitoring latex allergy for a number of years in the $\mathrm{UK}^{(9)}$. To date, however, there has not been any conclusive evidence that latex allergy has significantly increased, although recent reports have touched on this ${ }^{(10,11)}$. It is possible that the problem of latex sensitivity is under-reported in the UK.

Latex allergy may occur as a delayed hypersensitivity (type 4) reaction or immediate hypersensitivity (type 1) reaction. The immediate reaction is due to proteins present in the natural rubber latex and symptoms usually appear within 30 minutes of contact with latex. The main symptoms are urticaria and oedema, but if mucous membranes are affected then asthma, nasal congestion or conjunctivitis may occur. Rarely anaphylaxis may result, especially if the latex protein comes into contact with broken skin or mucous membranes. The delayed hypersensitivity reaction is predominantly caused by accelerating agents present in the rubber, introduced during the manufacturing process. This leads to allergic contact dermatitis of the skin and is characterised particularly by an erythematous or itching rash on the back of the hands. Vesicles or blisters may develop in severe reactions. A delayed reaction occurs several hours after contact with latex and is maximal after $24-48$ hours. It is important to distinguish between irritation due to cleansing agents and true latex allergy, and the risk of latex allergy is increased by the use of powdered gloves ${ }^{(12)}$. Although the modified starch powder itself is not an allergen, the protein residue present in latex readily attaches to starch which acts as a carrier or hapten to enhance the allergic potential. Therefore, the level of latex proteins available for sensitisation can be increased and, when gloves are taken off, the powder can be expressed in a cloud facilitating airborne transmission and absorption through mucous membranes. This can lead to asthma, itchy eyes or nasal congestion. Airborne powder provides a risk not only to the individual using the gloves but to other sensitised individuals working in the same environment. Furthermore, there is the possibility of sensitising previously unsensitised individuals from powder dispersal.

Individuals with atopy have a greater potential for allergy ${ }^{(13)}$ and are more likely to become latex-sensitive than non-atopic individuals. Furthermore, people with allergies to nuts or other agents need to be cautious since there is evidence of cross reactivity between various foods and latex allergens ${ }^{(14)}$.

Testing for latex allergy includes a skin test in which an aqueous extract of the rubber latex can be scratched or pricked into the skin and the resulting erythema and wheal can be compared with control agents. Otherwise, patch tests are performed: these involve a two-day occlusion of the test material to intact skin, and response is noted after 48-72 hours. Patch testing, however, could lead to sensitivity in previously non-sensitised individuals. In vitro testing (RAST or ELISA testing) is free of the latter complication and a simple blood test can detect specific latex $\operatorname{IgE}$ in allergic

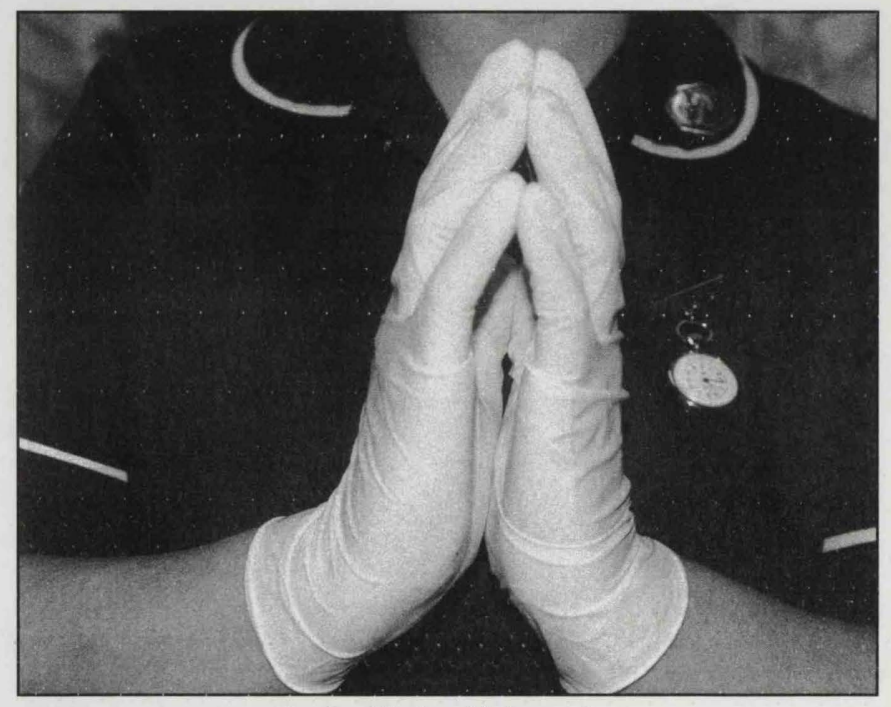

Fig. 3. - The humble latex glove 
individuals. No testing is absolute and a negative test does not necessarily exclude allergy completely.

Individuals allergic to latex should use latex-free gloves and two varieties are available: Allergard from Johnson and Johnson, and Dermaprene from Ansell Medical. Latex-free alternatives for other products, either for hospital or home use, are also available ${ }^{(11)}$.

It is relevant that $32 \%$ of 301 staff members who returned questionnaires did not know that latex glove allergy could be a potential problem. Furthermore $62 \%$ of the group did not know what procedures to follow if latex allergy was detected. Increased awareness of the problem is essential if we are to recognise the problems of latex allergy. Symptoms should not be dismissed as trivial and in each workplace there should be local knowledge of latex sensitivity and the availability of both powder-free and latex-free gloves should be mandatory in hospital environments. The present study has suggested that latex allergy is common in a population of hospital employees and is much greater than the incidence in the general population, which has been reported as less than $1 \%$, although higher in some risk groups ${ }^{(15)}$. Hospitals should develop protocols for dealing with latex-sensitive individuals and furthermore should be aware of potential problems that could arise in latex-sensitive patients requiring surgery.

\section{Acknowledgements}

We wish to thank all members of staff for their cooperation in this study, Sr Barbara Dean for supplying several articles on glove allergy and Julie Wood for typing the manuscript.

\section{REFERENCES}

1 Voelker R OSHA mandates universal precautions AMA News 16 December 1992

2 Buccino LA Latex sensitivity on increase (experts blame inferior rubber, exposure frequency) Advance for Medical Laboratory Professionals 1993;6-7

3 Allenius H, Makinen-Kiljunen S, Turjanmaa K, Palosuo T,
Reulana T Allergen and protein contact of latex gloves Ann Allergy 1994;73:315-210

4 Yassin MS, Leiri M, Fischer $\mathrm{T}$ et al Latex allergy in hospital employees Annals of Allergy 1994;72:245-249

5 Lagier F, Vervolet D, Lhermet I et al Prevalence of latex allergy in operating room nurses J Allergy Clin Immunol 1992;90:319

6 Beaudouin E, Pupil P, Moneret-Vautrin D et al Allergie professionelle au latex Rev Fr Allergol 1990;30:157-167

7 Turjanmaa K Incidence of immediate allergy to latex gloves in hospital personnel Contact Dermatitis 1987;17:270-275

8 Wrangsjo K, Osterman K, Van Hage-Hanisten M Gloverelated symptoms among operation theatre and dental care unit personnel Contact Dermatitis 1994;30:139-143

9 Latex sensitisation in the health care setting (use of latex gloves) Device Bulletin, Medical Devices Agency MDA DB 9601, April 1996

10 Booth B Hidden dilemma. Natural rubber (latex) allergy Nursing Times 1994;90:46-48

11 Bernstein M An overview of latex allergy and its implications for emergency nurses J Emer Nurs 1996;22:2936

12 Bauer X, Jager D Airbome antigens from latex gloves Lancet 1990;335:912

13 Moneret-Vautrin D, Beaudounin R, Widmer S et al Prospective study of risk factors in rubber latex hypersensitivity J Allergy Clin Immunol 1993;92:668-677

14 Rodruguez M, Vega F, Garcia MT. Hypersensitivity of latex, chestnut and banana. Ann Allergy 1993;70:31-34

15 Turjanmaa K, Reunala T Incidence of positive prick test to rubber protein. Contact Dermatitis 1990;23:279 